\title{
Ultrasound assisted particle and cell manipulation on-chip
}

\author{
Helen Mulvana ${ }^{a}$, Sandy Cochran ${ }^{b}$ and Martyn Hill ${ }^{c^{*}}$ \\ a School of Engineering, University of Glasgow, Glasgow G12 8QQ, United Kingdom \\ ${ }^{b}$ Institute for Medical Science and Technology, University of Dundee, DD2 1FD Dundee, United Kingdom \\ c Engineering Sciences and Institute for Life Sciences, University of Southampton, Southampton, United \\ Kingdom \\ * Corresponding author: Engineering Sciences, University of Southampton, Southampton, United Kingdom. Tel \\ +442380593075. E-mail address m.hill@soton.ac.uk
}

\section{ABSTRACT}

Ultrasonic fields are able to exert forces on cells and other micron-scale particles, including microbubbles. The technology is compatible with existing lab-on-chip techniques and is complementary to many alternative manipulation approaches due to its ability to handle many cells simultaneously over extended length scales. This paper provides an overview of the physical principles underlying ultrasonic manipulation, discusses the biological effects relevant to its use with cells, and describes emerging applications that are of interest in the field of drug development and delivery on-chip.

\section{INTRODUCTION}

Ultrasound is well known as a diagnostic imaging technique. However, its basis in pressure waves also allows it to be used to realise physical processes. In these, the role of the ultrasound is usually to transfer energy from a convenient location for its generation, through transduction of electrical to mechanical energy by piezoelectric material, to a location where it is applied. For example, ultrasound may be applied to the surface of the human body for non-invasive hyperthermic surgery [1], or it may be applied at a remote location in a lab-on-chip system to control or sort particles within a microfluidic channel [2].

Whilst ultrasound is sometimes utilised directly, e.g. to move small objects around through radiation force, a secondary transduction process is often utilised at the application location, e.g. through generation of cavitation bubbles [3] or heat, or through resonance of microbubbles to transform the energy in the wave into highly directed or otherwise localised fluid flow [4]. Approaches using microbubbles are discussed throughout the paper and covered in detail in section 4.3. This secondary transduction process provides a great deal of flexibility in its application and thus a very wide range of possible schemes and devices.

\subsection{Why is it relevant?}

Ultrasound has two separate domains of application relating to drug delivery. The first is as one of several techniques to manipulate cells and particles, including vehicles such as microbubbles for tasks such as drug discovery and delivery in microfluidic lab-on-chip devices [5], and the second is to mediate drug delivery in vivo, e.g. by applying local forces to tissues, which can enhance porosity, or to the drug delivery vehicles [6]. In both domains, ultrasound may also assist with the release of drugs from specific vehicles [7, 8]. As ultrasound has applications in both the laboratory and in vivo domains it has added-value for translation from the laboratory, the focus of this paper, to the clinical application. 
When considering microfluidic platforms, ultrasound can trap, levitate, and manipulate cells, large liposomes, and bubbles within a controlled environment. Further, ultrasonic fields can be used to construct cell aggregates and to increase mass transfer within the microfluidic environment. Thus ultrasound offers multiple opportunities to enhance on-chip functionality. In Section 2 we summarise the physical acoustic principles which may be harnessed within on-chip devices, while in Section 3 some key biological effects relevant to ultrasonic manipulation in vitro, including the potential to enhance or initiate drug delivery, are discussed. Several groups have demonstrated the capability of these techniques on-chip and such devices are discussed in Section 4 with particular emphasis on their applications in tissue engineering (4.2), and the study of drug-cell interactions for drug development and delivery (4.3).

\section{PHYSICAL PRINCIPLES}

Ultrasonic excitation within microfluidic systems typically leads to complex fields characterised by acoustic energy gradients of various length scales. These energy gradients underlie two phenomena of significance for the manipulation of cells and drug delivery vehicles such as microbubbles on chip: acoustic radiation forces and acoustic streaming. These are described in the following subsections prior to a discussion of the devices and typical parameters used in microfluidic ultrasonic manipulation.

\subsection{Radiation forces}

Cells within an ultrasonic field experience high frequency oscillatory forces due to the linear component of the acoustic field, but these forces have time-averages of zero that do not lead to translation of the cells. However there are second order terms within the field which have a finite time-average and generate acoustic radiation forces that can be used for steady state manipulation and levitation of cells and other particles. King [9] derived an expression for the acoustic radiation forces on a spherical, rigid particle in an in inviscid fluid and this analysis was extended by Yosioka and Kawasima [10] to allow for compressible particles and bubbles. Recently Bruus [11], following the approach of Gor'kov [12], developed a simple expression to allow for viscous effects. For the inviscid case [11] the radiation force $F_{a c}$ on a spherical particle of volume $V$ which is small in relation to the wavelength of a standing wave is:

$$
F_{a c}=-\nabla\left(V\left(f_{1} \frac{\kappa_{0}}{2}\left\langle p^{2}\right\rangle-f_{2} \frac{3 \rho_{0}}{4}\left\langle v^{2}\right\rangle\right)\right) .
$$

The time averages (represented by \langle\rangle ) of the squares of the acoustic pressure $p$ and velocity $v$ are proportional to the potential and kinetic energy densities of the standing wave field and it is the gradients of these energies that interact with the compressibilities $\left(\kappa_{0}, \kappa_{p}\right)$ and densities $\left(\rho_{0}, \rho_{p}\right)$ of the fluid and particle respectively. The coefficients $f_{1}$ and $f_{2}$ are given by

$$
f_{1}=1-\frac{\kappa_{p}}{\kappa_{0}} \text { and } f_{2}=\frac{2\left(\rho_{p}-\rho_{0}\right)}{2 \rho_{p}+\rho_{0}} .
$$

It can be seen that the gradient of the potential energy density interacts with the compressibilities of the particle and fluid to produce one component of the radiation force, while the gradient of the kinetic energy density interacts with the density difference between the particle and fluid to produce the other component of the force. Typically, the near-neutral buoyancy of cells and of some drug delivery vehicles means that the force is dominated by the pressure-compressibility interaction. Bruus [11] also demonstrated that the effect of 
viscosity on the radiation force can be represented by a correction to the coefficient $f_{2}$ and showed that the influence of viscosity is small if the density difference between the particle and fluid is small.

Depending on the geometry of the acoustic field these forces can be used to levitate cells and move them away from a surface, to trap cells against a flow [13], to push cells against a surface [14] or to bring cells together in a prescribed geometry [15]. The agglomeration of cells is modified by the existence of secondary radiation forces [16] in which the scattered field from one cell exerts a force on an adjacent cell. Such forces are only significant over short distances and generally act to draw cells together once they are in close proximity.

The coefficients $f_{1}$ and $f_{2}$ can each be either positive or negative depending on the relative compressibility and density respectively of the fluid and the particle. For particles such as cells and loaded liposomes which are slightly denser and less compressible than an aqueous medium, both coefficients are positive, tending to move cells to areas of low acoustic pressure amplitude (pressure nodes) and high acoustic velocity amplitude. However, less dense, more compressible particles will move to regions of high acoustic pressure amplitude and low acoustic velocity amplitude. This is the case for small bubbles, i.e. those that are driven at a frequency below their own acoustic resonance frequency. However larger bubbles, driven at a frequency that is higher than their resonance, will be forced towards a pressure node [10], as their scattered field changes phase above resonance. The acoustic radiation force on bubbles has been used to align, trap, and transport bubbles in micro-channels [17, 18], and to move bubbles to a target surface [19].

\subsection{Acoustic Streaming}

Gradients of acoustic energy density also lead to acoustic streaming: the acoustic generation of net fluid flows [20]. The two main sources of streaming are: i) due to acoustic energy dissipation into the bulk of a fluid, and ii) due to energy dissipation from the interaction of an acoustic field with a boundary. The first of these mechanisms, also known as "Eckart streaming", or the "quartz wind", is caused by attenuative loss over a number of wavelengths so is typically only an issue in microfluidic devices when high frequency waves propagate along the length of a channel [21]. It is also less likely to be observed in standing wave systems in which the forces leading to Eckart streaming from counter-propagating waves tend to cancel each other out. The second mechanism occurs due to steep energy gradients within the acoustic boundary layer adjacent to a wall ("boundary layer driven" streaming) or near the surface of an oscillating bubble ("cavitation microstreaming"), and these forms of streaming are widely observed in ultrasonically excited microfluidic devices. 


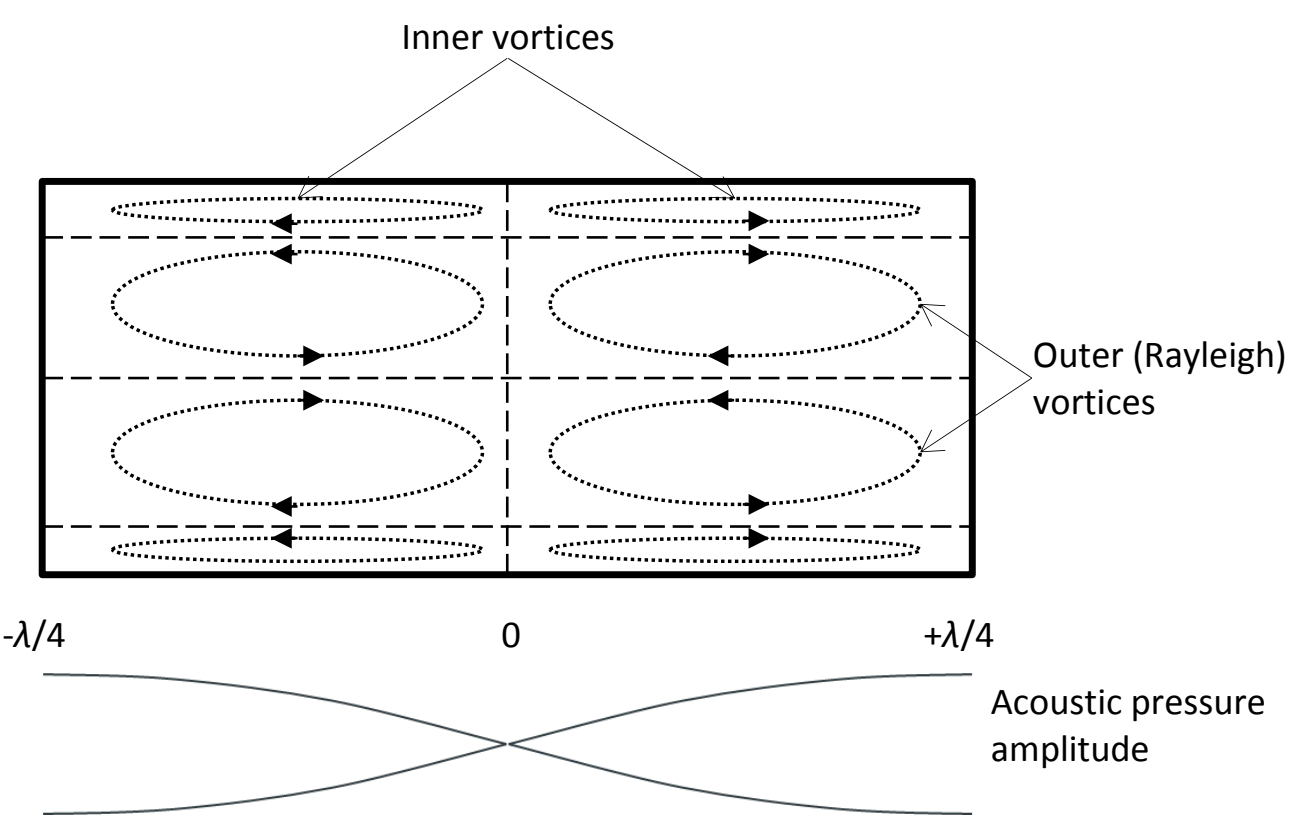

Figure 1. Schematic representation of streaming between two rigid walls (top and bottom) generated within an acoustic standing wave running left to right between two rigid reflectors, based on a figure included in [22].

Boundary layer driven streaming was first analysed by Rayleigh [23], describing the flow observed within a standing wave between parallel walls that leads to the four vortices within each half wavelength that characterises Rayleigh streaming (see Figure 1). Each of these four outer vortices is driven by an inner vortex generated by viscous losses within the viscous boundary layer. For a standing wave in water with a frequency in the low $\mathrm{MHz}$ range the wavelength will be of the order of $1 \mathrm{~mm}$, while the viscous boundary layer depth will be of the order of $1 \mu \mathrm{m}$ [21]. Hamilton et al. [22] show that for very narrow channels, Rayleigh's analysis ceases to be valid, and ultimately the outer streaming vortices cease to exist. More recently, Lei et al. [24] have shown that boundary driven streaming in microfluidic devices can occur in planes orthogonal to classical Rayleigh vortices.

Streaming can disrupt the ordered manipulation of cells via acoustic radiation forces as particles are drawn along the streaming vortices by the viscous drag forces. On large particles the radiation forces tend to dominate, while smaller particles are influenced primarily by the streaming drag. Bruus [25] has shown that for typical particles in a $1 \mathrm{MHz}$ standing wave in water the critical diameter below which streaming drag dominates is of the order of $2 \mu \mathrm{m}$. In other cases, the existence of streaming can be used to positive effect, improving mixing and mass transfer in biosensing applications [26], enhancing the interaction of cells and retroviruses [27], agglomerating cells in droplets [28] and the removal of non-specifically bound proteins from surfaces [29].

\subsection{Devices for on-chip ultrasonic manipulation}

A variety of different types of device have been used for ultrasonic manipulation [30]. Probably the simplest approach is to use a layered, or planar, resonator [31] comprising a transducer, which is typically isolated from the fluid in which the cells are suspended by a carrier, or coupling layer. The standing wave is maintained by a passive reflecting layer at the far side of the fluid, as shown in Figure 2. 


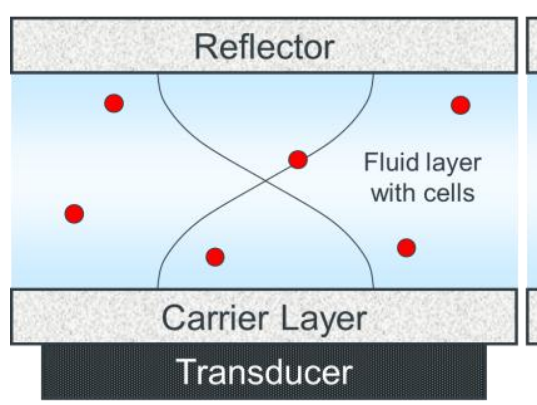

(a)

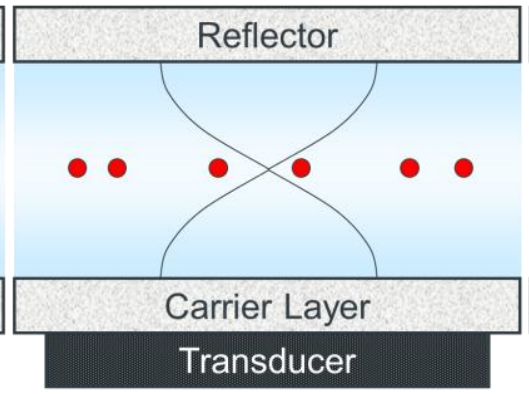

(b)

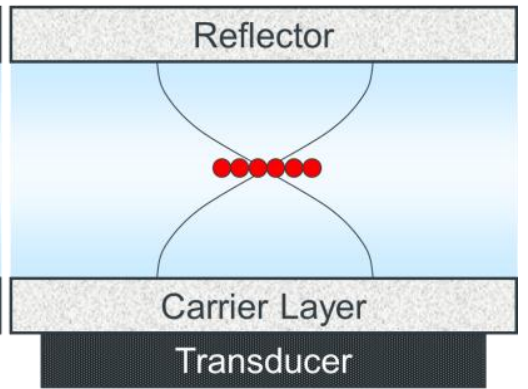

(c)

Figure 2. Schematic representation of layered resonator. A standing wave is set up by exciting the transducer at a resonant frequency which generates a pattern of pressure gradients within the fluid which effect the cell manipulation. Uniformly distributed cells (a), first move to a pressure node (b) and are then brought together within the node (c).

Such a resonator may be many wavelengths across, resulting in multiple planes into which cells are gathered [32]. Generally, the fluid layers in the microdevices considered here will be less than a wavelength thick and will gather cells into a single nodal plane [33,34]. An alternative arrangement, the transversal resonator, has been used extensively by Laurell's group in Lund [30,35]. In this arrangement the standing wave is established parallel to the transducer face such that manipulation operations can be easily observed through a glass cap above the channel and also parallel to the transducer face. Variations on these approaches can use glass capillaries [36] or a combination of planar and transversal excitation [37].

A rather different approach to the excitation of the ultrasonic field uses surface acoustic waves (SAWs) coupled into a micro-channel $[38,39]$. The SAWs are generated using interdigitated electrodes printed onto a piezoelectric substrate - typically lithium niobate - and generally the channel is fabricated from PDMS which is bonded on to the substrate. The use of transducer pairs allows a standing SAW to be established [40] which can be used to pattern particles or cells in one or two dimensions [41]. Using SAWs, significantly higher frequencies can be achieved (up to $150 \mathrm{MHz}$ ) allowing resolutions which can easily approach the single cell level, particularly when combined with focusing, which is typically achieved using phononic crystals [42]. SAWs can also be used to initiate fluid flow [39] and to provide energy (thermal and mechanical) for micro cell processing including PCR and cell lysis. A useful review of recent advances in SAW devices is presented by Wang et al. [43].

Recently there has been substantial interest in building devices that allow more dexterous manipulation of particles through the use of planar arrays of transducers [44] and different configurations of opposing transducers $[45,46]$.

\subsection{Limitations and typical parameters for on-chip manipulation}

Given the flexibility noted previously, and its potential for use in both lab-on-chip systems and in vivo, ultrasound has many useful potential capabilities. However, it also has several limitations. The first of these, shared with several other manipulation techniques, is the limited contrast between a fluid medium and cells or the particles (drug delivery vehicles for example) of interest in drug delivery [7]. In the case of ultrasound, this contrast is expressed in terms of the densities and compressibilities of the fluid and the particle, as discussed in section 2.1 above. Cells, micelles, and loaded drug delivery vehicles such as liposomes have parameters similar to those of water and tissue, limiting the efficiency of the ultrasound intervention. Relatively high ultrasound intensities are thus sometimes required to exert sufficient force, making secondary problems more likely. These include: acoustic streaming, which increases with increasing ultrasound intensity [20]; the need to control excessive heating of the lab-on-chip device through high drive signals applied to the piezoelectric source of ultrasound [47] and other materials in the structure; and cavitation [48], which occurs above a threshold depending on the level of the impurities in the fluid which act as cavitation nucleation sites and 
which may be expected to be high in lab-on-chip media. All these problems may affect cell viability [49]. On the other hand, gas-filled microbubbles have high ultrasound contrast because of their high compressibility [10]. This offers the possibility to label cells, to implement effective manipulation of drug delivery vehicles, and to manipulate various functional particles.

Another fundamental limitation of ultrasound is the difficulty to isolate it to specific regions within a substrate. Acoustic absorber materials are available commercially (e.g. Precision Acoustics, Dorchester, UK) but these may be difficult to integrate with other lab-on-chip fabrication processes. Ultrasound does not penetrate gaseous media effectively, so it may be possible to configure physical gaps in the device to contain propagation, but it is likely that ultrasound will propagate within the substrate. Thus, reports of arrays of ultrasound-actuated lab-on-chip devices on a common substrate focus on multiwall configurations [50] with ultrasound generated in a remote position on the substrate for application in the microfluidic components of a device, for example in transversal resonance [35] and SAW devices [38].

Acoustic isolation is a complicating factor in the fabrication of ultrasound-actuated lab-on-chip systems and the need for a piezoelectric material to generate ultrasound is another one. For SAW devices, the substrate is typically made of lithium niobate [51] and for bulk wave resonators, piezoelectric ceramic is used [52]. However, both of these are relatively expensive materials so they point towards the possibility to use a separate sterile microfluidic component, such as a capillary, to reduce the cost of disposal and replacement for each biological sample [30]. It is also possible to integrate piezoelectric materials in thick [53] or thin film [54, 55] form, or indeed bonded bulk material [56], with other components. Requiring an additional level of fabrication sophistication, typically including photolithography [57], this approach may be very useful when the number of devices is high and may be a key issue in differentiating acoustic manipulation from optical manipulation [58], where integration is still much harder.

As noted previously, another important issue is temperature. Ultrasound, in its nature as a wave phenomenon, involves propagation of local motion of material within the host solid or liquid media. (It does not propagate effectively in gas at the frequencies required for lab-on-chip use.) This motion is ultimately dissipated as heat, particularly in the solid components of the lab-on-chip system. Ingeniously, this is being harnessed in systems in which it is taken into account in the need to maintain the optimum temperature for cells [47]. In that case, it simply allows less external heating to be applied. However, care must be taken to avoid local hot-spots which may occur near the transducer because of excess heating from the ultrasound generation process or in relatively lossy media. For example, the acoustic attenuation (approximately equivalent to heating) in PDMS and other plastics is much higher than in glass and silicon [59].

Often, if adequate intensity can be achieved without causing damage, the most important parameter in ultrasound systems is frequency. Frequency is associated with several other properties of a lab-on-chip system, including the force that can be applied for given target moiety dimensions, as discussed in Section 2.1. The radiation force equations [12] suggest that, in general, higher frequencies should be used and as frequency relates inversely to wavelength [60], which defines the spatial resolution of the manipulation process, higher frequencies may also represent an improvement. For example, at frequency $f=1 \mathrm{MHz}$, the wavelength in water (and tissue, blood etc) $\lambda \approx 1.5 \mathrm{~mm}$. A comparison with typical dimensions of cells or drug delivery vehicles, of the order of $10 \mu \mathrm{m}$ or less, suggests that a higher frequency is indicated, for example, $\mathrm{f}=10 \mathrm{MHz}, \lambda \approx 150 \mu \mathrm{m}[61]$. Indeed, when SAW devices are adopted, in which higher frequencies are as easy to achieve as lower ones, frequencies of this order are used [62].

On the other hand, ultrasound frequency typically determines at least one dimension of the device used to generate it, for example thickness of piezoelectric material in bulk wave devices [52]. Thus, higher frequency operation almost inevitably complicates fabrication of thickness and transversal resonance devices [63, 64]. It also increases attenuation and thus heating, and acoustic streaming (see Section 2.2). The former may be beneficial in reducing leakage of ultrasound into unwanted regions in the device, but it is also likely to hinder 
scale-up. Thus, in general, where high frequency is easy to achieve, in SAW devices, it is used and where it is difficult and high resolution is desirable in bulk wave devices, the highest practical frequency is used. Nevertheless, there are many situations in which relatively low frequency bulk waves are used, even in relatively complicated devices; in this case, they are simply matched to the microfluidic channel dimensions [44].

\section{BIOLOGICAL EFFECTS}

In addition to the acoustic forces which can be generated using ultrasound for the purposes of particle manipulation, the management of acoustic forces to generate controlled bio-effects in cells and tissues provides an additional important role within on-chip devices; particularly for drug delivery applications. The two most important implications of these biological effects can be summarised as sonoporation, the generation of porosity in cell membranes using ultrasound and commonly used to enhance particle uptake in vivo for drug delivery, and cell viability, the sometimes undesired consequence of compromising cell viability as a result of the biological effects generated. These two issues are addressed here.

\subsection{Cell viability}

While ultrasound is well established as a medical tool for diagnostic imaging, it is also known to be capable of damaging tissue through thermal and mechanical effects [49], so if ultrasound is to be widely employed for the manipulation of cells it is important that potential effects on the cells themselves are investigated and understood. The frequencies and pressure amplitudes used in cell manipulation are of the same orders as those used in diagnostic imaging, and the fact that the bioeffects of imaging have been exhaustively studied without evidence of tissue damage suggests that cell damage in manipulation devices due to ultrasound is unlikely [65]. However the nature of the ultrasonic environment in a standing wave device is quite different from that experienced by cells in tissue during imaging so a thorough evaluation of the response of different cell types to such fields is crucial [65]. Studies by Doblhoffdier et al. [66] suggested that there was no significant loss in the viability of mammalian cells suspended in a large ultrasonic resonator at operating power levels. This conclusion was also reached in a study by Bohm et al. [67] using a similar chamber. These authors noted a significant difference between the response of cells to standing and travelling waves of similar frequency and pressure amplitude and a review of the behaviour of cells in such large resonators under different operating conditions was provided by Shirgaonkar et al. [68]. The viability of mammalian cells held by ultrasonic forces within a mesh has been shown to be retained [69] and erythrocytes exposed to acoustic field strengths high enough to induce mixing showed no significant damage [69]. Hultström et al. [70] suspended aggregates of cells in a flowing culture medium within a microfluidic chip and showed that not only their viability, but also their ability to proliferate was maintained and the same group demonstrated that cells exposed to ultrasound in a multi-well micro-plate for three days retained the potential to proliferate [50]. A series of papers by Bazou and co-workers has examined the behaviour of ultrasonically suspended cell aggregates [71, 72] and have shown that embryonic stem cells levitated in an ultrasonic field for an hour retain pluripotency and their gene expression is unmodified [73]. It should be noted however, that while ultrasound at the frequencies and pressures employed in these studies (low MHz regime and 0.08-0.85 MPa) was not shown to influence viability or proliferation, other studies have shown that ultrasound is capable of modifying cell surfaces through removal of the microvillae normally present on them [74].

\subsection{Sonoporation in resonant chambers}

While the use of ultrasound-driven microbubbles to enhance the delivery of materials to cells has largely dominated the literature, sonoporation in the absence of microbubbles has perhaps remained underexplored. Nevertheless, ultrasound alone has also been demonstrated as an effective method of improving cellular uptake of genes [75], plasmid [76] and fluorescent probes [77]. In fact a number of studies have demonstrated effective delivery using ultrasound alone and indicate that such techniques can be conducted with good cell 
viability, which in some cases may present an advantage over microbubble techniques which can lead to cell death and capillary damage under some operating conditions.

Lee et al. [75], used ultrasound standing waves in the absence of microbubbles to overcome the low transduction efficiency commonly associated with retrovirus delivery to cells in vitro resulting in a four-fold improvement in delivery. Erythroleukemia cells arranged in bands using standing waves generated at $1 \mathrm{MHz}$ (continuous wave) were exposed to circulating vesicular retroviruses. The small viruses (100 nm) were attracted to these bands where the cells acted as nucleating sites, improving the encounter probability between cells and delivery material. The effect was enhanced by the size disparity between cells and retroviruses, since acoustic radiation force dominates over microstreaming for large particles leading to the ability to agglomerate cells, while the reverse is true for smaller ones allowing the circulation of retroviruses amongst the cells. The group were able to demonstrate that using this technique uptake was improved over Brownian motion alone as it was hypothesised that cells were exposed to the retroviruses before their rapid decay.

The study claimed that the effect of sonoporation could be ruled out from having an effect on improving cell delivery because exposure to travelling waves in the absence of standing waves did not improve cellular uptake. However, since sonoporation simply means an increase in cell permeability due to sound and the impact of microstreaming, which other studies have demonstrated may generate cell permeability [78], has not been established in this study this claim may not be entirely accurate. Finally, the authors point out that use of ultrasound standing waves leads to high rates of cell viability and improves the suitability of ultrasound to enhance delivery of materials to cell suspensions and not just plated cell lines, making it particularly applicable to large scale applications.

Rodamporn et al.[76] similarly found transfection of HeLa cells was improved through exposure to ultrasound standing waves, while maintaining cell viability in excess of $78 \%$. In this case cells were transfected with a plasmid encoding green fluorescent protein using a sonoporation frequency ( $980 \mathrm{KHz}, 0-19.5 \mathrm{MPa}$ ) determined through impedance analysis of the fluid layer contained within a custom designed chamber, incorporating fluid inlet and outlets and a piezoceramic active element and matching layer operating around a glass chamber. In this case it was hypothesised that successful delivery of material to cells occurred as a result of sonoporation generated by the viscous shearing occurring as a result of microstreaming.

A further study employing the techniques presented by Rodamporn et al. found two population effects when considering the porosity developed in cells exposed to standing waves at $2.27 \mathrm{MHz}$ over a pressure range from 0.11-1.39 MPa [77]. The first population of cells showed an exponential decay in porosity over time following the initial exposure. It was theorised that this could have occurred as a result of the initial acoustic exposure used to initiate transport to the pressure antinode, since it has been theorised that sonoporation may result from intramembraneous cavitation [79]. The second population of cells showed sustained poration over a longer period. In this case the authors hypothesise that poration only occurred following exposure of these cells to a critical number of oscillatory strain cycles. In summary the authors believe poration may result from a combination of these effects as evidenced by the two distinct populations and may be further enhanced by the fluid shear stresses generated by non-inertial streaming.

\section{EXAMPLE APPLICATIONS}

\subsection{Overview}

Ultrasonic manipulation has been investigated in a variety of microfluidic applications. Two recent review papers distinguish between applications that involve the trapping of particles and cells [13] and those that use radiation forces within continuous flow microsystems [2]. Trapping of particles can be used to enhance 
bioassays [80], by increasing bead interactions in agglutination assays [81], or by trapping particles on functionalised surfaces [14]. Cells can be trapped directly in order to concentrate them for forensic analysis [36] and it is possible to enhance the trapping of small bacteria and nanoparticles by introducing larger seed particles into the ultrasonic field [82].

Success in ultrasonic manipulation at the single cell level has also been demonstrated [83]. A rather different approach has enabled single cells to be trapped and manipulated by very high frequency focused ultrasound. While fabrication challenges are commonly cited as the limiting factor in using single element focused ultrasound devices to manipulate cells, recent progress in piezoelectric materials generation and handling indicate the situation may be changing. Lee et al., [84] recently demonstrated the manipulation of a single 10 $\mu \mathrm{m}$ leukaemia cell using a focused transducer operating at $200 \mathrm{MHz}$. The device consisted of a $6 \mu \mathrm{m}$ thick layer of zinc oxide sputtered on the back surface of a sapphire buffer rod, formed at the opposing surface to generate a spherical focusing lens. Acoustic coupling into water was improved through addition of a glass matching layer. The resulting device had a beam full width at half maximum of $9.5 \mu \mathrm{m}$ at its focus, $0.5 \mathrm{~mm}$ from the front face of the focusing lens.

Lenshoff et al. [2] describe continuous flow systems for effecting concentration, filtration, separation, characterisation, medium-exchange, and the switching of particles between streams for applications such as fluorescence-activated cell sorting. These applications are described extensively in [2] and other recent reviews $[31,43]$ so the remainder of this section will concentrate on the applications relevant to tissue engineering and drug-cell interactions.

\subsection{Assembly and Tissue Engineering}

The ability of ultrasonic fields to control the position of cells suggests that it should be possible to use the technology to enhance the formation of cell aggregates for in vitro tissue engineering, prior to implantation for example. Further, for certain cell types there is evidence that the microenvironment provided by ultrasonic excitation can modify and enhance the production of the cells' extra cellular matrix [85, 86]. A detailed study of the response of chondrocytes to culture within polymer scaffolds under ultrasonic excitation [87] observed positive changes in viability, proliferation, and gene expression, and modified expression of a large number of proteins, in comparison with control samples in the absence of ultrasonic excitation. One relevant application of ultrasound is for enhancing the seeding of tissue engineering scaffolds. Surface acoustic waves can be used to generate acoustic streaming within a cell-containing droplet, to drive that droplet to a porous scaffold, and then use the acoustically induced flow to improve the penetration of the cells within the scaffold $[88,89]$.

Working without scaffolds, Liu et al. [90] formed aggregates of a hepatocyte cell line within an ultrasonic trap, using the ultrasound to accelerate the aggregate formation and to modify their geometry during the initial 30 minutes of their formation. The cells were then maintained in culture in the absence of ultrasound for up to three weeks and shown to have similar functional characteristics to hepatocyte spheroids prepared using gyrotation culture techniques. The same group subsequently modified the technique so as to culture the cells within alginate beads following ultrasonically assisted formation [91] and further demonstrated the ultrasonic assembly of multiple cell aggregates simultaneously within different geometry resonators [92]. More recently, Bazou et al. [15] extended the techniques for the controlled assembly of two and three dimensional suspended cell aggregates by demonstrating that changing the characteristics of a pulsed standing wave could be used to modify the geometry of the aggregates.

Garvin et al. have used ultrasonic fields to pattern cells within collagen hydrogels [86] and suggest that the ultrasound-induced cell patterning itself has a significant influence on the structure of the extracellular matrix. They later used this technique to organise endothelial cells so as to form a vascular-like bed within the matrix [93]. Mazzoccoli et al. also worked in hydrogels to form concentric cylindrical arrangements of a breast cancer cell line (MDA-MB231) [94]. 
An alternative approach relies on the potential of multiple pairs of transducers to create one and two dimensional patterns of particles or cells. Ding et al. [95] arranged lines of stromal cells and hepatocytes at controlled positions on a substrate using standing surface acoustic waves. Bernassau et al. [96] used bulk acoustic waves generated by a heptagonal arrangement of transducers to act as an ultrasonic stencil, to deposit predefined patterns of cells on a glass cover slip and also demonstrated the ability to place different batches of cells at predefined relative positions. The group has also demonstrated the ability to pattern functionalised microspheres and microbubbles with the same technology [97]. Finally, the use of ultrasound transducer arrays holds significant promise for adaptive systems in which cell positions are monitored, allowing the electronic signals driving the array to be adjusted as the cell cultures develop. This is illustrated in simple form by an adaptation of the configuration shown in Fig. 2 to include a one-dimensional transducer array as shown in Fig. 3.

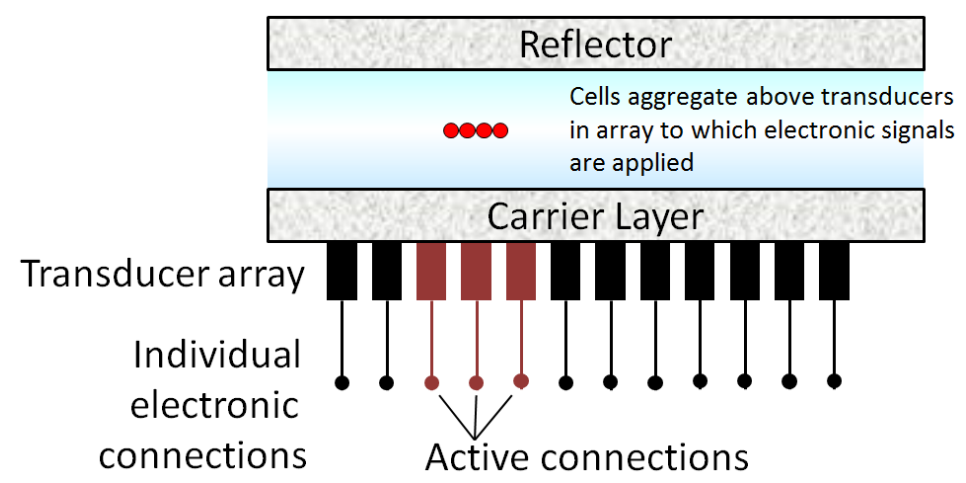

Figure 3. An extension of the arrangement in Fig. 2 in which the single transducer beneath the cells is replaced by a transducer array under electronic control [44]. This allows the device to adapt its behaviour in the lateral direction according to changes in the cell culture. For example, two aggregates of cells could be grown separately under levitation then brought together at an appropriate stage.

\subsection{Study of drug-cell interaction}

Ultrasound can be used in lab-on-chip configurations to enhance the application of drugs to both cell ensembles and single cells, unlike some alternative techniques such as optical tweezing [98] which are appropriate mainly for single cells. In addition, its realisation with piezoelectric devices [30] allows relatively straightforward integration with other lab-on-chip components, principally those used to fabricate microfluidic channels and systems. Amongst the most exciting work at present is in single cell manipulation, and it is on this that we concentrate here. Despite the significant advances in lab-on-chip technologies and their application to devices for drug delivery systems, the goal of single cell manipulation and the ability to perform automated tasks on single cells remains a challenge. The application of ultrasound, and the several technologies embodied by ultrasound, to this rapidly developing field presents several important opportunities in the pursuit of singlecell level manipulation within lab-on-chip devices.

The control, processing and analysis of single cells are essential for the continuing development of on-chip drug delivery systems. The ability to operate on single cells rather than cell populations limits dilution of the cell content, improving process efficiency, control and sensitivity. It allows the processing and analysis of several samples simultaneously to produce large volumes of detailed information on cellular responses, and enables improved dosage and experimental control [99]. Effective trapping, isolation and sorting of single cells will enable targeted delivery for gene therapy applications, detailed study of drug-cell interactions in drug delivery and development, cell selection for IVF, and improved sensitivity as required to address several emerging diagnostic applications [100]. These requirements are emphasised further by improved communitywide appreciation of the issues relating to cell heterogeneity and the need to investigate how the resulting stochastic and transient variations in cellular response can impact drug development and treatment efficiency. 
Until recently, two approaches could be considered to dominate contactless single cell/particle manipulation, negative dielectrophoresis (electrical forces) and laser tweezing (optical forces). Each offers high spatial resolution and accuracy but also requires complicated and costly equipment. Ultrasonic cell manipulation may offer the potential to address many of the challenges outlined above, particularly when considered alongside microfluidic and microplate manipulation. Ultrasound holds benefits over competing technologies since it can be achieved at relatively low cost without significant equipment investment. It is highly suited to incorporation within optical microscopy, and employs relatively low power densities for improved cell viability and opportunities for longer term cell study [70].

There are many examples of inclusion within microdevices with the aim of particle manipulation for on-chip technologies. Motivated by the time-consuming nature of generating statistically significant data for cell interactions within in vitro suspensions, Forslund et al demonstrated that ultrasound can be used to induce and synchronise cell-cell contact between natural killer (NK) and target cells in multiwall plates [101]. The microchip platform developed was compatible with microscopy analysis and supported coordination of multiple experiments simultaneously.

\section{Enhancement of ultrasound effects using gas microbubbles}

Particular success in ultrasound mediated drug delivery has been achieved with the use of gas microbubbles [102-105]. The disparity in acoustic properties between the gas and surrounding media present several opportunities to augment the effects of ultrasound alone. Microbubbles, consisting of a high molecular weight gas core stabilised by a lipid, protein or polymer shell, were first produced commercially as ultrasound contrast agents (USCAs) in the 1980s [106]. Their ability to act as foci for the release of ultrasound energy has long been established and has led to their exploitation for therapeutic applications [102, 107, 108]. The huge interest generated by the technique supports a vibrant field of research and significant literature concerning characterisation now exists [109-111].

Upon excitation by an acoustic wave, microbubbles undergo rapid expansion and contraction [112]. This can be used to generate stable cavitation and localised shear stresses at low acoustic intensities [113]. As the acoustic intensity increases the microbubble shell can no longer support the increasing radial excursion leading to fragmentation. At high acoustic intensities, microbubbles can be driven to inertial cavitation resulting in catastrophic collapse and gas jetting. Their strongly resonant behaviour allows the extent of the effects to be manipulated through ultrasound frequency selection and their non-linear response, particularly at higher acoustic intensities, supports the generation of acoustic radiation forces. Fig. 4 shows an example of albumincoated microbubble oscillation under acoustic excitation leading to shell disruption and gas jetting. 

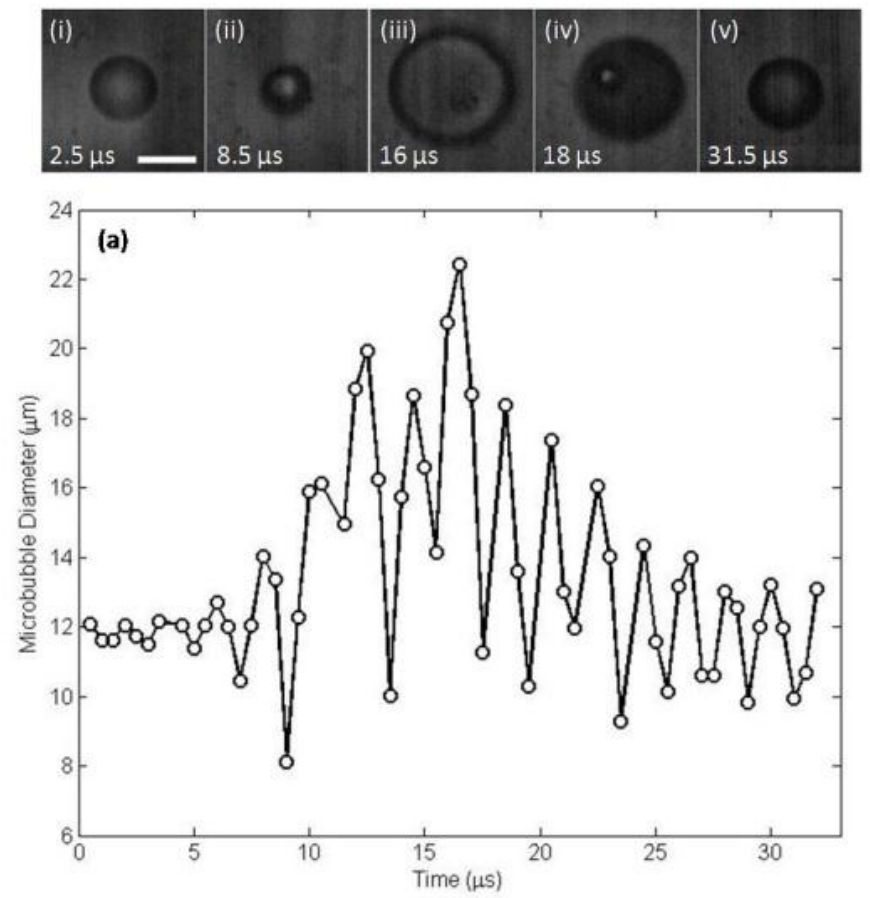

Figure 4. Change in microbubble diameter during ultrasound excitation $(0.5 \mathrm{MHz}$ carrier signal windowed using a Gaussian function with a full width half maximum equivalent to 12 cycles of the carrier and peak negative pressure of approximately $100 \mathrm{kPa}$ ) (a) and a series of sample images used to produce the trace, illustrating the microbubble at its (i) initial, (ii) maximum and (iii) minimum diameter, (iv) evidence of gas jetting and (v) final diameter. The high speed images were acquired at a camera frame rate of $2 \mathrm{Mfps}$. (Reproduced from [114] with permission from Elsevier).

Microbubbles can be used to generate transient porosity in cells and vasculature at diagnostic ultrasound intensities to facilitate drug and gene delivery both in vitro and in vivo $[115,116]$. It is generally considered that both stable and inertial cavitation lead to the generation of bio-effects which either mechanically rupture the phospholipid structure of the cell membrane or disrupt/invoke cell-regulated pathways. In each case, it is accepted that the resulting pores are resealed by the cell within a few minutes; thus this approach is considered to be less destructive at a cellular level than alternative methods such as electroporation, laser irradiation and microinjection for cellular transfection [102].

As microbubbles can be functionalised with targeting ligands to improve their affinity to particular tissues or disease locations [117] they can transport drugs or genes for ultrasound mediated release at a particular location [103, 118, 119]. In micro devices, microbubbles have been used for selective trapping based on particle size and density before further processing (mixing and aggregation) [120]. The layout of the device developed for this follows the basic principle illustrated in Figure 2.

A significant limitation of microbubbles in vivo is their rapid clearance from the blood pool as they are either destroyed by ultrasound, dissolve due to local changes in the content and gradient of dissolved blood-gases, are mechanically disrupted as they pass through the cardiac and pulmonary systems or are sequestered by the reticuloendothelial system. In microfluidic devices, the limitations are likely to involve high surface tension, shear stresses, rapid changes of pressure or exposure to high pressure, and friction generated heating and evaporation of the small volumes of water involved; these remain to be overcome before microbubble utilisation in micro devices can be considered optimum.

Finally, in addition to the employment of microbubbles in on-chip devices, novel techniques for microbubble fabrication [121] have been adapted to miniaturised platforms [122, 123] enabling lab-on-chip microbubble fabrication for drug delivery applications and the control of microbubble diameter to yield monodisperse microbubble suspensions. The techniques have also been demonstrated for the fabrication of liposome 
functionalised microbubbles [122] and in some cases the intention is that this sort of technology could be used to deliver microbubbles from an intrasvascular catheter directly to the treatment location [123].

A key driver in many drug delivery investigations is to determine the mechanisms responsible for porosity generation in order to improve delivery efficiencies when such techniques are applied in vivo. In addition to the well-recognised involvement of cavitation, recent studies have demonstrated an association between oscillating microbubbles and some physiological or cell regulated mechanisms of porosity, or, for example, endocytosis $[124,125]$.

\section{Drug Discovery}

A significant potential application of ultrasound for particle and cell manipulation is within the arena of drug discovery, where exhaustive in vitro investigation of drug - cell interactions is an essential step in understanding and developing new therapeutic agents. The additional control offered by incorporating ultrasound manipulation techniques within existing experimental protocols offers significant opportunities to accelerate early stage drug development. The manipulation techniques outlined above provide the means to coordinate delivery of materials across multiwell plates [50] for parallel investigation and aggregate drugs, cells or combinations of each to control and initiate interactions at specific time points. The results could improve experimental throughput by orders of magnitude reducing development times and perhaps enabling more comprehensive investigations. It is also likely that such powerful in vitro development tools could lead to reduced in vivo experimental requirements, which can be unpopular and expensive. Indeed, several investigators have highlighted the relevance of their approaches to drug discovery [76, 77] and Fig. 5 shows the approach taken by Carugo et al. [77] to enhance drug uptake in the absence of contrast agent.

a
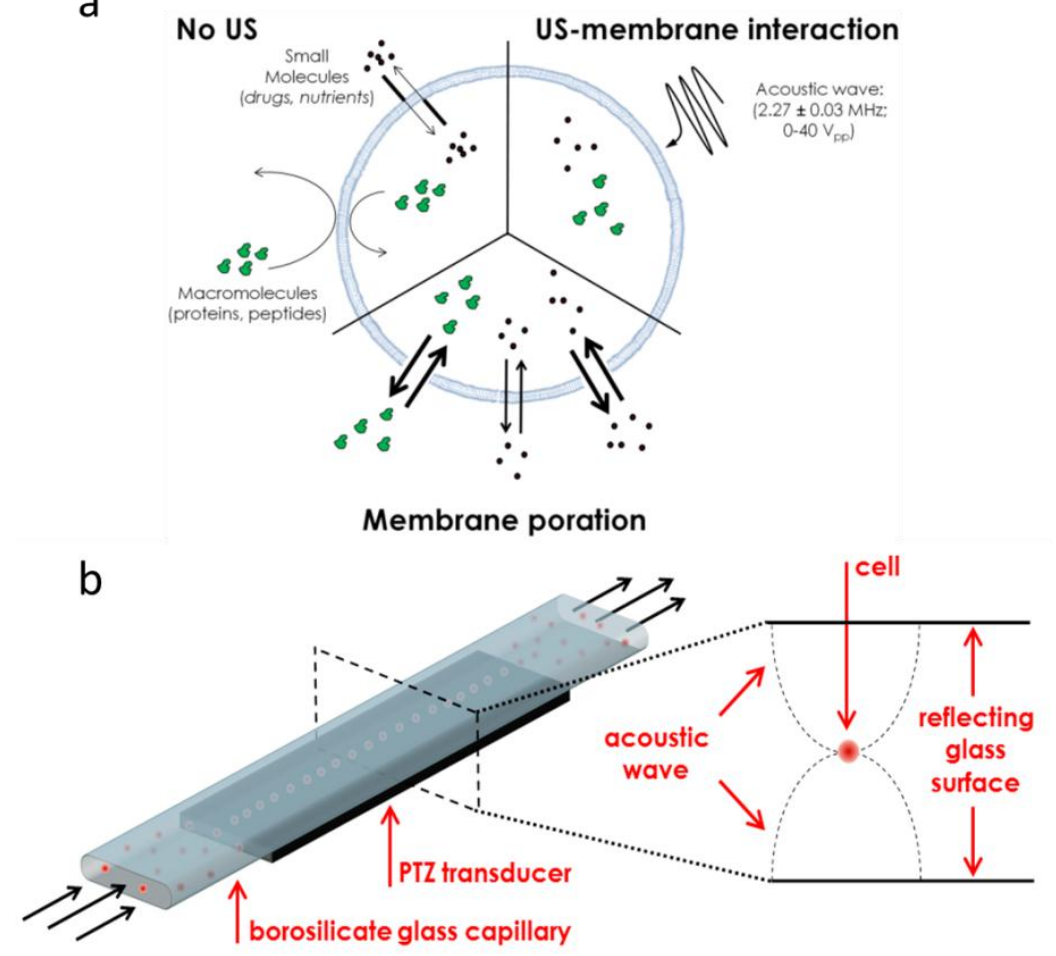

Figure 5. Schematic representation of sonoporation as described by Carugo et al. [77]. (a) In the absence of ultrasound the cell membrane is permeable only to small molecules (top-left). Under ultrasonic excitation (top right) transmembrane transfer of molecules was facilitated (bottom). (b) Sonoporation took place within an ultrasonic standing wave excited within a capillary, which moved cells to the acoustic pressure node. Reprinted with permission from Carugo et al. [77]. Copyright 2011, American Institute of Physics. 
Potential of ultrasound for drug delivery

The influence of ultrasound on both cells and carriers for drugs, linked with its established ability to penetrate into tissue without any incisions, has led to significant research in ultrasound-mediated drug delivery [126]. In turn this suggests two directions for on-chip ultrasound-assisted work, first, to take advantage of the same capabilities that make ultrasound attractive for drug delivery and, second, to minimise the barriers in translation from research and development in vitro to application in vivo.

Use of ultrasound to deliver materials across the normally impermeable cell membrane is well documented $[99,127]$. In these applications it is thought that acoustic cavitation is the dominant mechanism, where dissolved gases are caused to nucleate and then rapidly collapse at high peak negative acoustic intensities, releasing energy and free radicals and causing jetting [128]. As mentioned above, standing waves have also been demonstrated to be effective in enhancing cellular uptake $[76,78,129]$ where the mechanism is thought to be microstreaming, resulting in shear stresses on the cell membrane leading to porosity. Recent studies have suggested that standing wave generation may be as important as cavitation in effective ultrasound mediated drug delivery [78] and that it can have a protective effect, improving cell viability, due to the absence of a greatly fluctuating pressure [130].

Ultrasound can also affect drug carriers and thus offers two routes to drug delivery, the first by increasing the permeability of cells so that the drug-carrier complex can enter the cells for drug release there, with chemical / biological processes, and the second by enhancing the release of the drug from its carrier in proximity to the target cells through heating or mechanical disruption, potentially with cavitation. For ultrasound-mediated delivery, carriers can take the form of complex molecules [131], micelles and liposomes [132], and microbubbles [133]. Molecular carriers, micelles and liposomes are particularly amenable to the first mechanism but microbubbles are too big. However, as microbubbles loaded with drugs are closely equivalent to conventional ultrasound contrast agents, mechanisms are available to break them down into pieces small enough to penetrate biological systems including vascular walls and cells [105].

It is thus possible to foresee a translational pathway in which ultrasound-mediated drug delivery is investigated first in vitro with lab-on-chip approaches based on existing and future acoustic manipulation techniques [131], followed by a conventional in vivo pathway through small animals to human trials based on established and future ultrasound beam forming algorithms, potentially taking advantage of advanced techniques adapted from optics, such as the use of Bessel beams [134]. Equipment specifically for this translation pathway is already under development, such as the high throughput sonicator system shown in Fig. 6 and reported in detail elsewhere [135].

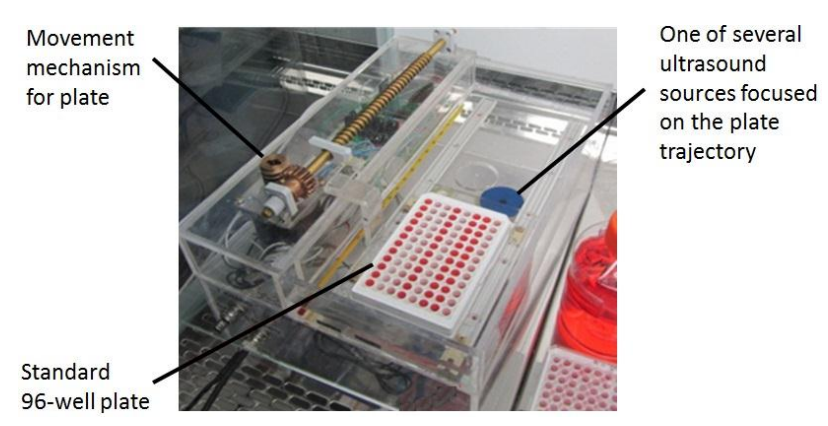

Figure 6. A system designed to allow high throughput evaluation of the effects of ultrasound for drug delivery. The standard 96-well plate is passed over several ultrasound transducers under computer control, allow selective sonication of the contents of particular wells.

\section{CONCLUSIONS AND OUTLOOK FOR THE FUTURE}

Ultrasound offers a means of developing controlled, non-contact forces capable of extending the functionality of lab-on-chip devices. In many cases ultrasound offers advantages over current methods used on-chip. For example, the use of acoustic tweezers for particle manipulation and sorting rather than optical tweezers offers 
advantages in cost, space requirements, and the use of regulated equipment (high intensity lasers), while exerting minimal impact on cell viability and proliferation [65]. There is documented evidence that standing waves can generate cellular stress [78] and further work is required to understand the implications of this. Ultrasound can be used with microbubbles to extend functionality to include mixing [120], mechanical excitation of cells [113], drug delivery [102], and spatial and temporal control of drug release [103, 119]. Ultrasound may be considered to be most effective as a means of manipulating groups of cells or particles. This provides significant potential for building and investigating tissue constructs on-chip and provides a useful tool in exploring cell-cell interaction [101]. Devices with more dexterous manipulation abilities [46, 97] should be able to extend this capability, for example by allowing different cell types to be brought together in a highly controlled environment. There is also potential to use acoustic manipulation in conjunction with other force fields such as optical tweezers or dielectrophoresis that have a more local scale of action [136], taking advantage of the strengths of both approaches. In most cases further work is required before ultrasound alone can be used effectively to manipulate single cells or other micron-scale particles, however, this may well be a realistic possibility in future devices [84].

\section{REFERENCES}

[1] J. Wu, W.L.M. Nyborg, Emerging therapeutic ultrasound, World Scientific, Hackensack, N.J., 2006. [2] A. Lenshof, C. Magnusson, T. Laurell, Acoustofluidics 8: Applications of acoustophoresis in continuous flow microsystems, Lab on a Chip, 12 (2012) 1210-1223.

[3] E.A. Neppiras, Acoustic Cavitation, Physics Reports-Review Section of Physics Letters, 61 (1980) 159-251.

[4] R. Manasseh, P. Tho, A. Ooi, K. Petkovic-Duran, Y. Zhu, Cavitation microstreaming and material transport around microbubbles, in: L.G. Garreton (Ed.) International Congress on Ultrasonics, Proceedings, 2010, pp. 427-432.

[5] M.A. Sobanski, C.R. Tucker, N.E. Thomas, W.T. Coakley, Sub-micron particle manipulation in an ultrasonic standing wave: Applications in detection of clinically important biomolecules, Bioseparation, 9 (2000) 351-357.

[6] J.P. Kilroy, A.L. Klibanov, B.R. Wamhoff, J.A. Hossack, Intravascular Ultrasound Catheter to Enhance Microbubble-Based Drug Delivery via Acoustic Radiation Force, IEEE Trans. Ultrason. Ferroelectr. Freq. Control, 59 (2012) 2156-2166.

[7] K.W. Ferrara, M.A. Borden, H. Zhang, Lipid-Shelled Vehicles: Engineering for Ultrasound Molecular Imaging and Drug Delivery, Accounts of Chemical Research, 42 (2009) 881-892.

[8] A. van Wamel, K. Kooiman, M. Harteveld, M. Emmer, F.J. ten Cate, M. Versluis, N. de Jong, Vibrating microbubbles poking individual cells: Drug transfer into cells via sonoporation, Journal of Controlled Release, 112 (2006) 149-155.

[9] L.V. King, On the acoustic radiation pressure on spheres, Proc R. Soc. London, A147 (1934) 212240.

[10] K. Yosioka, Y. Kawasima, Acoustic radiation pressure on a compressible sphere, Acustica, 5 (1955) 167-173.

[11] H. Bruus, Acoustofluidics 7: The acoustic radiation force on small particles, Lab on a Chip, 12 (2012) 1014-1021.

[12] L.P. Gor'kov, On the forces acting on a small particle in an acoustical field in an ideal fluid, Sov. Phys. Dokl., 6 (1962) 773-775.

[13] M. Evander, J. Nilsson, Acoustofluidics 20: Applications in acoustic trapping, Lab on a Chip, 12 (2012) 4667-4676. 
[14] P. Glynne-Jones, R.J. Boltryk, M. Hill, F. Zhang, L. Dong, J.S. Wilkinson, T. Brown, T. Melvin, N.R. Harris, Multi-modal particle manipulator to enhance bead-based bioassays, Ultrasonics, 50 (2010) 235-239.

[15] D. Bazou, A. Castro, M. Hoyos, Controlled cell aggregation in a pulsed acoustic field, Ultrasonics, 52 (2012) 842-850.

[16] M. Gröschl, Ultrasonic separation of suspended particles - Part I: Fundamentals, Acustica, 84 (1998) $432-447$.

[17] L. Meng, F. Cai, Q. Jin, L. Niu, C. Jiang, Z. Wang, J. Wu, H. Zheng, Acoustic aligning and trapping of microbubbles in an enclosed PDMS microfluidic device, Sensors and Actuators B: Chemical, 160 (2011) 1599-1605.

[18] L. Meng, F. Cai, Z. Zhang, L. Niu, Q. Jin, F. Yan, J. Wu, Z. Wang, H. Zheng, Transportation of single cell and microbubbles by phase-shift introduced to standing leaky surface acoustic waves, Biomicrofluidics, 5 (2011) 044104.

[19] X. Xi, F.B. Cegla, M. Lowe, A. Thiemann, T. Nowak, R. Mettin, F. Holsteyns, A. Lippert, Study on the bubble transport mechanism in an acoustic standing wave field, Ultrasonics, 51 (2011) 10141025.

[20] S. Boluriaan, P. Morris, Acoustic streaming: from Rayleigh to today, International Journal of Aeroacoustics, 2 (2003) 255-292.

[21] M. Wiklund, R. Green, M. Ohlin, Acoustofluidics 14: Applications of acoustic streaming in microfluidic devices, Lab on a Chip, 12 (2012) 2438-2451.

[22] M.F. Hamilton, Y.A. Ilinskii, E.A. Zabolotskaya, Acoustic streaming generated by standing waves in two-dimensional channels of arbitrary width, The Journal of the Acoustical Society of America, 113 (2003) 153-160.

[23] Lord Rayleigh, On the Circulation of Air Observed in Kundt's Tubes and on Some Allied Acoustical Problems, Philosophical Transactions, 175 (1884) 1-21.

[24] J. Lei, P. Glynne-Jones, M. Hill, Acoustic streaming in the transducer plane in ultrasonic particle manipulation devices, Lab on a Chip, 13 (2013) 2133-2143.

[25] H. Bruus, Acoustofluidics 10: Scaling laws in acoustophoresis, Lab on a Chip, 12 (2012) 15781586.

[26] L.A. Kuznetsova, W.T. Coakley, Applications of ultrasound streaming and radiation force in biosensors, Biosensors \& Bioelectronics, 22 (2007) 1567-1577

[27] Y.H. Lee, J.O. You, C.A. Peng, Retroviral transduction of adherent cells in resonant acoustic fields, Biotechnol. Prog., 21 (2005) 372-376.

[28] H. Li, J.R. Friend, L.Y. Yeo, Surface acoustic wave concentration of particle and bioparticle suspensions, Biomedical Microdevices, 9 (2007) 647-656.

[29] R. Singh, S.K.R.S. Sankaranarayanan, V.R. Bhethanabotla, Enhancement of acoustic streaming induced flow on a focused surface acoustic wave device: Implications for biosensing and microfluidics, Journal Of Applied Physics, 107 (2010) 024503.

[30] A. Lenshof, M. Evander, T. Laurell, J. Nilsson, Acoustofluidics 5: Building microfluidic acoustic resonators, Lab on a Chip, 12 (2012) 684-695.

[31] P. Glynne-Jones, R.J. Boltryk, M. Hill, Acoustofluidics 9: Modelling and applications of planar resonant devices for acoustic particle manipulation, Lab on a Chip, 12 (2012) 1417-1426.

[32] J.J. Hawkes, S. Radel, Acoustofluidics 22: Multiwavelength devices and scale dependent properties, Lab on a Chip, In press (2013).

[33] J.J. Hawkes, W.T. Coakley, Force field particle filter, combining ultrasound standing waves and laminar flow, Sens. Actuator B-Chem., 75 (2001) 213-222.

[34] N.R. Harris, M. Hill, S.P. Beeby, Y. Shen, N.M. White, J.J. Hawkes, W.T. Coakley, A Silicon Microfluidic Ultrasonic Separator, Sens. Actuator B-Chem, 95 (2003) 425-434.

[35] F. Petersson, A. Nilsson, C. Holm, H. Jonsson, T. Laurell, Separation of lipids from blood utilizing ultrasonic standing waves in microfluidic channels, Analyst, 129 (2004) 938-943. 
[36] B. Hammarstrom, M. Evander, H. Barbeau, M. Bruzelius, J. Larsson, T. Laurell, J. Nilsson, Noncontact acoustic cell trapping in disposable glass capillaries, Lab on a Chip, 10 (2010) 2251-2257.

[37] O. Manneberg, J. Svennebring, H.M. Hertz, M. Wiklund, Wedge transducer design for twodimensional ultrasonic manipulation in a microfluidic chip, Journal of Micromechanics And Microengineering, 18 (2008) 095025.

[38] M. Gedge, M. Hill, Acoustofluidics 17: Theory and applications of surface acoustic wave devices for particle manipulation, Lab on a Chip, 12 (2012) 2998 - 3007.

[39] L.Y. Yeo, J.R. Friend, Ultrafast microfluidics using surface acoustic waves, Biomicrofluidics, 3 (2009) 012002

[40] J.J. Shi, H. Huang, Z. Stratton, Y.P. Huang, T.J. Huang, Continuous particle separation in a microfluidic channel via standing surface acoustic waves (SSAW), Lab on a Chip, 9 (2009) 3354-3359.

[41] C.D. Wood, J.E. Cunningham, R. O'Rorke, C. Walti, E.H. Linfield, A.G. Davies, S.D. Evans, Formation and manipulation of two-dimensional arrays of micron-scale particles in microfluidic systems by surface acoustic waves, Appl. Phys. Lett., 94 (2009) 054101.

[42] J. Reboud, Y. Bourquin, R. Wilson, G.S. Pall, M. Jiwaji, A.R. Pitt, A. Graham, A.P. Waters, J.M. Cooper, Shaping acoustic fields as a toolset for microfluidic manipulations in diagnostic technologies, Proceedings of the National Academy of Sciences of the United States of America, 109 (2012) 1516215167.

[43] Z.C. Wang, J.A. Zhe, Recent advances in particle and droplet manipulation for lab-on-a-chip devices based on surface acoustic waves, Lab on a Chip, 11 (2011) 1280-1285.

[44] P. Glynne-Jones, C. Démoré, C. Ye, Y. Qiu, S. Cochran, M. Hill, Array Controlled Ultrasonic Manipulation of Particles in Planar Acoustic Resonator, IEEE Transactions on Ultrasonics, Ferroelectrics and Frequency Control, 59 (2012) 1258-1266.

[45] A. Grinenko, C.K. Ong, C.R.P. Courtney, P.D. Wilcox, B.W. Drinkwater, Efficient counterpropagating wave acoustic micro-particle manipulation, Appl. Phys. Lett., 101 (2012) 233501.

[46] A. Grinenko, P.D. Wilcox, C.R.P. Courtney, B.W. Drinkwater, Proof of principle study of ultrasonic particle manipulation by a circular array device, Proc. R. Soc. A-Math. Phys. Eng. Sci., 468 (2012) 3571-3586.

[47] M. Evander, L. Johansson, T. Lilliehorn, J. Piskur, M. Lindvall, S. Johansson, M. Almqvist, T. Laurell, J. Nilsson, Noninvasive acoustic cell trapping in a microfluidic perfusion system for online bioassays, Analytical Chemistry, 79 (2007) 2984-2991.

[48] T.G. Leighton, The Acoustic Bubble, Academic Press Inc., 1997.

[49] W.L. Nyborg, Biological effects of ultrasound: Development of safety guidelines. Part II: General review, Ultrasound Med. Biol., 27 (2001) 301-333.

[50] B. Vanherberghen, O. Manneberg, A. Christakou, T. Frisk, M. Ohlin, H.M. Hertz, B. Onfelt, M. Wiklund, Ultrasound-controlled cell aggregation in a multi-well chip, Lab on a Chip, 10 (2010) 27272732.

[51] K.K. Wong, Properties of Lithium Niobate, in, IET, 2002.

[52] A. Safari, E.K. Akdogan, Piezoelectric and Acoustic Materials for Transducer Applications, Springer, 2008.

[53] N.R. Harris, M. Hill, R.N. Torah, R.J. Townsend, S.P. Beeby, N.M. White, J. Ding, A Multilayer Thick-film PZT Actuator for MEMs Applications, Sensors and Actuators A: Physical, 132 (2006) 311316.

[54] P. Muralt, Recent progress in materials issues for piezoelectric MEMS, Journal of the American Ceramic Society, 91 (2008) 1385-1396.

[55] P. Muralt, R.G. Polcawich, S. Trolier-McKinstry, Piezoelectric Thin Films for Sensors, Actuators, and Energy Harvesting, Mrs Bulletin, 34 (2009) 658-664.

[56] E.E. Aktakka, R.L. Peterson, K. Najafi, Wafer-Level Integration of High-Quality Bulk Piezoelectric Ceramics on Silicon, IEEE Trans. Electron Devices, 60 (2013) 2022-2030.

[57] J.H. Sweet, D. Hutson, S. Cochran, A. Bernassau, Concepts and issues in piezo-on-3D silicon structures, Sensor Review, 29 (2009) 326-332. 
[58] S. Cochran, A.L. Bernassau, D.R.S. Cumming, C.E.M. Demore, M.P.Y. Desmulliez, J. Sweet, Future integration of silicon electronics with miniature piezoelectric ultrasonic transducers and arrays, in: IEEE International Ultrasonics Symposium, IEEE, San Diego, 2010, pp. 1108 - 1116.

[59] K. Zell, J.I. Sperl, M.W. Vogel, R. Niessner, C. Haisch, Acoustical properties of selected tissue phantom materials for ultrasound imaging, Physics in Medicine and Biology, 52 (2007) N475-N484.

[60] R.S.C. Cobbold, Foundations of Biomedical Ultrasound, Oxford University Press USA, 2006.

[61] V. Yantchev, J. Enlund, I. Katardjiev, L. Johansson, A micromachined Stoneley acoustic wave system for continuous flow particle manipulation in microfluidic channels, Journal Of Micromechanics And Microengineering, 20 (2010) 035031.

[62] L. Johansson, J. Enlund, S. Johansson, I. Katardjiev, V. Yantchev, Surface acoustic wave induced particle manipulation in a PDMS channel-principle concepts for continuous flow applications, Biomedical Microdevices, 14 (2012) 279-289.

[63] Y. Qiu, C. Demore, S. Sharma, S. Cochran, D.A. Hughes, K. Weijer, Multi-wavelength ultrasonic standing wave device for non-invasive cell manipulation and characterisation, in: Ultrasonics Symposium (IUS), 2011 IEEE International, 2011, pp. 188-191.

[64] K.H. Lam, H.-S. Hsu, Y. Li, C. Lee, A. Lin, Q. Zhou, E.S. Kim, K.K. Shung, Ultrahigh frequency lensless ultrasonic transducers for acoustic tweezers application, Biotechnology and Bioengineering, 110 (2013) 881-886.

[65] M. Wiklund, Acoustofluidics 12: Biocompatibility and cell viability in microfluidic acoustic resonators, Lab on a Chip, 12 (2012) 2018-2028.

[66] O. Doblhoffdier, T. Gaida, H. Katinger, W. Burger, M. Groschl, E. Benes, A Novel Ultrasonic Resonance Field Device for the Retention of Animal-Cells, Biotechnol. Prog., 10 (1994) 428-432.

[67] H. Bohm, P. Anthony, M.R. Davey, L.G. Briarty, J.B. Power, K.C. Lowe, E. Benes, M. Groschl, Viability of plant cell suspensions exposed to homogeneous ultrasonic fields of different energy density and wave type, Ultrasonics, 38 (2000) 629-632.

[68] I.Z. Shirgaonkar, S. Lanthier, A. Kamen, Acoustic cell filter: a proven cell retention technology for perfusion of animal cell cultures, Biotechnology Advances, 22 (2004) 433-444.

[69] Z.W. Wang, P. Grabenstetter, D.L. Feke, J.M. Belovich, Retention and viability characteristics of mammalian cells in an acoustically driven polymer mesh, Biotechnol. Prog., 20 (2004) 384-387.

[70] J. Hultström, O. Manneberg, K. Dopf, H.M. Hertz, H. Brismar, M. Wiklund, Proliferation and viability of adherent cells manipulated by standing-wave ultrasound in a microfluidic chip, Ultrasound Med. Biol., 33 (2006) 175-181.

[71] D. Bazou, W.T. Coakley, K.M. Meek, M. Yang, D.T. Pham, Characterisation of the morphology of 2-D particle aggregates in different electrolyte concentrations in an ultrasound trap, Colloid Surf. APhysicochem. Eng. Asp., 243 (2004) 97-104.

[72] D. Bazou, G.P. Dowthwaite, I.M. Khan, C.W. Archer, J.R. Ralphs, W.T. Coakley, Gap junctional intercellular communication and cytoskeletal organization in chondrocytes in suspension in an ultrasound trap, Molecular Membrane Biology, 23 (2006) 195-205.

[73] D. Bazou, R. Kearney, F. Mansergh, C. Bourdon, J. Farrar, M. Wride, Gene Expression Analysis of Mouse Embryonic Stem Cells Following Levitation in an Ultrasound Standing Wave Trap, Ultrasound Med Biol, 37 (2011) 321-330.

[74] C.L. Kerr, D.W. Gregory, M. Shammari, D.J. Watmough, D.N. Wheatley, Differing effects of ultrasound-irradiation on suspension and monolayer cultured hela cells, investigated by scanning electron microscopy, Ultrasound in medicine \& biology, 15 (1989) 397-401.

[75] Y.H. Lee, C.A. Peng, Enhanced retroviral gene delivery in ultrasonic standing wave fields, Gene therapy, 12 (2005) 625-633.

[76] S. Rodamporn, N.R. Harris, S.P. Beeby, R.J. Boltryk, T. Sanchez-Elsner, HeLa Cell Transfection Using a Novel Sonoporation System, IEEE Transactions on Biomedical Engineering, 58 (2011) 927934. 
[77] D. Carugo, D.N. Ankrett, P. Glynne-Jones, L. Capretto, R.J. Boltryk, X. Zhang, P.A. Townsend, M. Hill, Contrast agent-free sonoporation: the use of an ultrasonic standing wave microfluidic system for the delivery of pharmaceutical agents, Biomicrofluidics, 5 (2011) 044108-044115.

[78] M.A. Hassan, M.A. Buldakov, R. Ogawa, Q.L. Zhao, Y. Furusawa, N. Kudo, T. Kondo, P. Riesz, Modulation control over ultrasound-mediated gene delivery: Evaluating the importance of standing waves, J. Control. Release, 141 (2010) 70-76.

[79] B. Krasovitski, V. Frenkel, S. Shoham, E. Kimmel, Intramembrane cavitation as a unifying mechanism for ultrasound-induced bioeffects, Proc. Natl. Acad. Sci. U. S. A., 108 (2011) 3258-3263.

[80] M. Wiklund, S. Radel, J.J. Hawkes, Acoustofluidics 21: ultrasound-enhanced immunoassays and particle sensors, Lab on a Chip, 13 (2013) 25-39.

[81] M. Wiklund, H.M. Hertz, Ultrasonic enhancement of bead-based bioaffinity assays, Lab on a Chip, 6 (2006) 1279-1292.

[82] B. Hammarstrom, T. Laurell, J. Nilsson, Seed particle-enabled acoustic trapping of bacteria and nanoparticles in continuous flow systems, Lab on a Chip, 12 (2012) 4296-4304.

[83] O. Manneberg, B. Vanherberghen, J. Svennebring, H.M. Hertz, B. Onfelt, M. Wiklund, A threedimensional ultrasonic cage for characterization of individual cells, Applied Physics Letters, 93 (2008) 063901.

[84] J. Lee, C. Lee, H.H. Kim, A. Jakob, R. Lemor, S.Y. Teh, A. Lee, K.K. Shung, Targeted Cell Immobilization by Ultrasound Microbeam, Biotechnology and Bioengineering, 108 (2011) 16431650.

[85] N.P. Whitney, A.C. Lamb, T.M. Louw, A. Subramanian, Integrin-mediated mechanotransduction pathway of low-intensity continuous ultrasound in human chondrocytes, Ultrasound Med. Biol., 38 (2012) 1734-1743.

[86] K.A. Garvin, D.C. Hocking, D. Dalecki, Controlling the Spatial Organization of Cells and Extracellular Matrix Proteins in Engineered Tissues Using Ultrasound Standing Wave Fields, Ultrasound Med Biol, 36 (2010) 1919-1932.

[87] A. Subramanian, J.A. Turner, G. Budhiraja, S.G. Thakurta, N.P. Whitney, S.S. Nudurupati, Ultrasonic Bioreactor as a Platform for Studying Cellular Response, Tissue Eng. Part C-Methods, 19 (2013) 244-255.

[88] H. Li, J.R. Friend, L.Y. Yeo, A scaffold cell seeding method driven by surface acoustic waves, Biomaterials, 28 (2007) 4098-4104.

[89] M. Bok, H.Y. Li, L.Y. Yeo, J.R. Friend, The Dynamics of Surface Acoustic Wave-Driven Scaffold Cell Seeding, Biotechnology And Bioengineering, 103 (2009) 387-401.

[90] J. Liu, L.A. Kuznetsova, G.O. Edwards, J. Xu, M. Ma, W.M. Purcell, S.K. Jackson, W.T. Coakley, Functional three-dimensional HepG2 aggregate cultures generated from an ultrasound trap: Comparison with HepG2 spheroids, JOURNAL OF CELLULAR BIOCHEMISTRY, 102 (2007) 1180-1189.

[91] D. Bazou, W.T. Coakley, A.J. Hayes, S.K. Jackson, Long-term viability and proliferation of alginate-encapsulated 3-D HepG2 aggregates formed in an ultrasound trap, Toxicology in Vitro, 22 (2008) 1321-1331.

[92] L.A. Kuznetsova, D. Bazou, G.O. Edwards, W.T. Coakley, Multiple Three-Dimensional Mammalian Cell Aggregates Formed Away from Solid Substrata in Ultrasound Standing Waves, Biotechnol. Prog., 25 (2009) 834-841.

[93] K.A. Garvin, D. Dalecki, D.C. Hocking, Vascularization of Three-Dimensional Collagen Hydrogels Using Ultrasound Standing Wave Fields, Ultrasound in Medicine \&amp; Biology, 37 (2011) 18531864.

[94] J.P. Mazzoccoli, D.L. Feke, H. Baskaran, P.N. Pintauro, Development of multilayered cellhydrogel composites using an acoustic focusing technique, Biotechnol. Prog., 26 (2010) 600-605.

[95] X. Ding, J. Shi, S.-C.S. Lin, S. Yazdi, B. Kiraly, T.J. Huang, Tunable patterning of microparticles and cells using standing surface acoustic waves, Lab on a Chip, 12 (2012) 2491-2497.

[96] A.L. Bernassau, F. Gesellchen, P.G.A. MacPherson, M. Riehle, D.R.S. Cumming, Direct patterning of mammalian cells in an ultrasonic heptagon stencil, Biomedical Microdevices, 14 (2012) 559-564. 
[97] A.L. Bernassau, P.G.A. MacPherson, J. Beeley, B.W. Drinkwater, D.R.S. Cumming, Patterning of microspheres and microbubbles in an acoustic tweezers, Biomedical Microdevices, 15 (2013) 289297.

[98] K. Dholakia, W.M. Lee, Optical Trapping Takes Shape: The Use of Structured Light Fields, in: Arimondo, et al. (Eds.) Advances In Atomic, Molecular, and Optical Physics, Academic Press, 2008, pp. 261-337.

[99] D.L. Miller, C. Dou, Induction of apoptosis in sonoporation and ultrasonic gene transfer, Ultrasound Med. Biol., 35 (2009) 144-154.

[100] D. Di Carlo, H.T.K. Tse, D.R. Gossett, Introduction: why analyze single cells?, Methods in molecular biology (Clifton, N.J.), 853 (2012) 1-10.

[101] E. Forslund, K. Guldevall, P.E. Olofsson, T. Frisk, A.E. Christakou, M. Wiklund, B. Onfelt, Novel Microchip-Based Tools Facilitating Live Cell Imaging and Assessment of Functional Heterogeneity within NK Cell Populations, Frontiers in immunology, 3 (2012) 300.

[102] S. Hernot, A.L. Klibanov, Microbubbles in ultrasound-triggered drug and gene delivery, Advanced Drug Delivery Reviews, 60 (2008) 1153-1166.

[103] A.L. Klibanov, T.I. Shevchenko, B.I. Raju, R. Seip, C.T. Chin, Ultrasound-triggered release of materials entrapped in microbubble-liposome constructs: A tool for targeted drug delivery, Journal of Controlled Release, 148 (2010) 13-17.

[104] R. Deckers, C.T.W. Moonen, Ultrasound triggered, image guided, local drug delivery, Journal of Controlled Release, 148 (2010) 25-33.

[105] K. Ferrara, R. Pollard, M. Borden, Ultrasound microbubble contrast agents: Fundamentals and application to gene and drug delivery, Annual Review of Biomedical Engineering, 9 (2007) 415-447.

[106] S.B. Feinstein, P.A. Heidenreich, C.D. Dick, J.M. Schneider, A.F. Pastoret, W.A. Rubenstein, J. Applebaum, J.L. Brehm, S. Aronson, J. Ellis, et al., Albunex a new intravascular ultrasound contrast agent preliminary safety and efficacy results, Circulation, 78 (1988) II565-II565.

[107] M. Postema, A. van Wamel, F.J. ten Cate, N. de Jong, High-speed photography during ultrasound illustrates potential therapeutic applications of microbubbles, Medical Physics, 32 (2005) 3707-3711.

[108] A. Hashmi, G. Yu, M. Reilly-Collette, G. Heiman, J. Xu, Oscillating bubbles: a versatile tool for lab on a chip applications, Lab on a Chip, 12 (2012) 4216-4227.

[109] H. Mulvana, E. Stride, J.V. Hajnal, R.J. Eckersley, Temperature Dependent Behavior of Ultrasound Contrast Agents, Ultrasound in medicine \& biology, 36 (2010) 925-934.

[110] V. Sboros, Response of contrast agents to ultrasound, Advanced Drug Delivery Reviews, 60 (2008) 1117-1136.

[111] J. Sijl, E. Gaud, P.J.A. Frinking, M. Arditi, N. de Jong, D. Lohse, M. Versluis, Acoustic characterization of single ultrasound contrast agent microbubbles, J. Acoust. Soc. Am., 124 (2008) 4091-4097.

[112] T.G. Leighton, The Acoustic Bubble, Academic Press, San Diego, 1994.

[113] R. Green, P. Glynne-Jones, R.J. Boltryk, P. Townsend, M. Hill, Controlling non-inertial cavitation microstreaming for applications in biomedical research, in: International Congress on Ultrasonics Gdansk, Poland, 2011, pp. 749-752.

[114] H. Mulvana, R.J. Browning, M.-X. Tang, J.V. Hajnal, R.J. Eckersley, Albumin Coated Microbubble Optimization: Custom Fabrication and Comprehensive Characterization, Ultrasound in Medicine \& Biology, 38 (2012) 1599-1607.

[115] A. Rahim, S.L. Taylor, N.L. Bush, G.R. ter Haar, J.C. Bamber, C.D. Porter, Physical parameters affecting ultrasound/microbubble-mediated gene delivery efficiency in vitro, Ultrasound in medicine \& biology, 32 (2006) 1269-1279.

[116] S. Mehier-Humbert, F. Yan, P. Frinking, M. Schneider, R.H. Guy, T. Bettinger, Ultrasoundmediated gene delivery: Influence of contrast agent on transfection, Bioconjugate Chem., 18 (2007) 652-662. 
[117] J.J. Rychak, J.R. Lindner, K. Ley, A.L. Klibanov, Deformable gas-filled microbubbles targeted to P-selectin, Journal of Controlled Release, 114 (2006) 288-299.

[118] B. Geers, I. Lentacker, N.N. Sanders, J. Demeester, S. Meairs, S.C. De Smedt, Self-assembled liposome-loaded microbubbles: The missing link for safe and efficient ultrasound triggered drugdelivery, Journal of Controlled Release, 152 (2011) 249-256.

[119] A.F.H. Lum, M.A. Borden, P.A. Dayton, D.E. Kruse, S.I. Simon, K.W. Ferrara, Ultrasound radiation force enables targeted deposition of model drug carriers loaded on microbubbles, Journal of Controlled Release, 111 (2006) 128-134.

[120] P. Rogers, A. Neild, Selective particle trapping using an oscillating microbubble, Lab on a Chip, 11 (2011) 3710-3715.

[121] U. Farook, H.B. Zhang, M.J. Edirisinghe, E. Stride, N. Saffari, Preparation of microbubble suspensions by co-axial electrohydrodynamic atomization, Medical Engineering \& Physics, 29 (2007) 749-754.

[122] S.A. Peyman, R.H. Abou-Saleh, J.R. McLaughlan, N. Ingram, B.R.G. Johnson, K. Critchley, S. Freear, J.A. Evans, A.F. Markham, P.L. Coletta, S.D. Evans, Expanding 3D geometry for enhanced onchip microbubble production and single step formation of liposome modified microbubbles, Lab on a Chip, 12 (2012) 4544-4552.

[123] A.J. Dixon, A.H. Dhanaliwala, J.L. Chen, J.A. Hossack, Enhanced Intracellular Delivery of a Model Drug Using Microbubbles Produced by a Microfluidic Device, Ultrasound in Medicine \& Biology, 39 (2013) 1267-1276.

[124] M. Afadzi, S. Strand, E. Nilssen, S.-E. Masoy, T. Johansen, R. Hansen, B. Angelsen, C. de L Davies, Mechanisms of the ultrasound-mediated intracellular delivery of liposomes and dextrans, IEEE transactions on ultrasonics, ferroelectrics, and frequency control, 60 (2013) 21-33.

[125] B.D.M. Meijering, L.J.M. Juffermans, A. van Wamel, R.H. Henning, I.S. Zuhorn, M. Emmer, A.M.G. Versteilen, W.J. Paulus, W.H. van Gilst, K. Kooiman, N. de Jong, R.J.P. Musters, L.E. Deelman, O. Kamp, Ultrasound and Microbubble-Targeted Delivery of Macromolecules Is Regulated by Induction of Endocytosis and Pore Formation, Circ.Res., 104 (2009) 679-U226.

[126] S. Mitragotri, Innovation - Healing sound: the use of ultrasound in drug delivery and other therapeutic applications, Nat. Rev. Drug Discov., 4 (2005) 255-260.

[127] V. Frenkel, Ultrasound mediated delivery of drugs and genes to solid tumors, Advanced Drug Delivery Reviews, 60 (2008) 1193-1208.

[128] S. Mehier-Humbert, R.H. Guy, Physical methods for gene transfer: Improving the kinetics of gene delivery into cells, Advanced Drug Delivery Reviews, 57 (2005) 733-753.

[129] S. Tinkov, G. Winter, C. Coester, R. Bekeredjian, New doxorubicin-loaded phospholipid microbubbles for targeted tumor therapy: Part I - Formulation development and in-vitro characterization, Journal of Controlled Release, 143 (2010) 143-150.

[130] M. Wiklund, B. Onfelt, Ultrasonic manipulation of single cells, Methods in molecular biology (Clifton, N.J.), 853 (2012) 177-196.

[131] D. Gourevich, Y. Hertzberg, A. Volovick, Y. Shafran, G. Navon, S. Cochran, A. Melzer, Ultrasound-Mediated Targeted Drug Delivery Generated by Multifocal Beam Patterns: An Invitro Study, Ultrasound in medicine \& biology, 39 (2013) 507-514.

[132] J.W. Yoo, N. Doshi, S. Mitragotri, Adaptive micro and nanoparticles: Temporal control over carrier properties to facilitate drug delivery, Advanced Drug Delivery Reviews, 63 (2011) 1247-1256.

[133] J. Castle, M. Butts, A. Healey, K. Kent, M. Marino, S.B. Feinstein, Ultrasound-mediated targeted drug delivery: recent success and remaining challenges, Am. J. Physiol.-Heart Circul. Physiol., 304 (2013) H350-H357.

[134] C.E.M. Demore, Z. Yang, A. Volovick, S. Cochran, M.P. MacDonald, G.C. Spalding, Mechanical Evidence of the Orbital Angular Momentum to Energy Ratio of Vortex Beams, Physical Review Letters, 108 (2012) 194301. 
[135] B. Gerold, D. Gourevich, D. Xu, F. Arditti, P. Prentice, S. Cochran, J. Gnaim, M. Yoav, L. Wang, A. Melzer, Applicator for In-vitro Ultrasound-activated Targeted Drug Delivery, in: Proc. 11th Int. Symp. Therapeutic Ultrasound 2012, pp. 100 - 105.

[136] P. Glynne-Jones, M. Hill, Acoustofluidics 23: Acoustic manipulation combined with other force fields, Lab on a Chip, 13 (2013) 1003-1010. 